\title{
Modelling and computation of large-scale open atmosphere hydrogen- air deflagration
}

\author{
Mohamed Sakr \\ HySAFER, School of Built Environment, Ulster University, Jordanstown Campus, \\ BT370QB, UK \\ sakr-m@ulster.ac.uk
}

\begin{abstract}
Studying of gas deflagration is important for a safety purpose in gas industry. A modelling approach based on large eddy simulation (LES) technique for modelling turbulent flow combined with the species mass fraction equations for modelling combustion is used. Different flame acceleration mechanisms, hydrodynamic \& thermo-diffusive instabilities, turbulence, and their interaction in addition to flame quenching model are used to model chemical reaction rate. An algebraic model for flame-generated turbulence is incorporated.
\end{abstract}

The model is tested against large scale open atmosphere hydrogen-air experiment. The flame propagation radius and the overpressures are qualitatively compared well with experiment and the state-of-the-art simulations.

Keywords: computational fluid dynamics, turbulence closure, combustion closure

\section{Introduction}

It is important to study gas deflagration for a safety purpose in gas industry, so that a mitigation technique could be applied in case of an accidental scenario. Computation of gas deflagration would require resolving turbulent flow and combustion. Three techniques are used to resolve turbulent flow based on the percentage of the resolved turbulent spectrum. For fully resolved spectrum, the technique is called Direct Numerical Simulation (DNS). For fully modelling of the turbulent spectrum, the technique is called Reynolds-Average- NavierStokes (RANS). For partially resolving of the spectrum, the technique is called Large Eddy Simulation (LES). Given that, the industrial geometries are in the order of 100 meters. The DNS approach is not possible using the current computational power for studying industrial scales.

Computation of combustion would require resolving chemical reactions with multi-species, which requires high computational resources. Different 
alternative approaches could be used to overcome this difficulty. The progress variable equation is one of these techniques. It is based on using a scalar with the value from 0 to 1 , where 0 represents no combustion and 1 represents full combustion. The intermediate values present the progress of the combustion. The main effort would be to model the chemical reaction rate (source term of the progress variable equation).

The current work is based on using LES technique to model turbulent flow and the combustion product species mass fraction equation, like the progress variable equation, to model combustion.

\section{Modelling approach}

The conservation equations of the mass, momentum, energy, and species mass fraction are resolved in their filtered form using the LES technique. The closure of the turbulence model is achieved based on filtered dynamic turbulent kinetic energy equation. A flame-generated turbulence model is incorporated as an algebraic source term to the momentum equation.

Heat of chemical reaction (source term of the energy equation) and chemical reaction rate (source term of the species mass fraction equations) are combustion-based terms. They are modelled based on a flame acceleration concept, where different acceleration, mechanisms hydrodynamic \& thermodiffusive instabilities, turbulence, and their interaction are incorporated. A possibility of flame quenching due to excessive stretching of the flame is also incorporated. A detail description of the equations is given in [1].

\section{Testing of the model}

The present section presents numerical simulation using the proposed chemical reaction rate model against large-scale hydrogen-air deflagration experiment in the open atmosphere. The open atmosphere experiment was used as a numerical benchmark for developing computational fluid dynamics (CFD) codes for a safety purpose.

Different teams have simulated the open atmosphere experiment in [2], [3]. They have used different combustion and turbulence modelling as shown in Table 1 [3]. 
Table 1: Turbulence and combustion models of the SOTA of the open atmosphere [3]

\begin{tabular}{|l|l|l|}
\hline Team & Turbulence model & Combustion model \\
\hline CEA & None & CREBCOM Combustion model \\
\hline FzK & RANS standard $(k-\varepsilon)$ & CREBCOM model with flame tracking \\
\hline Gexon & RANS standard $(k-\varepsilon)$ & Beta flame model \\
\hline NH & RANS standard $(k-\varepsilon)$ & Beta flame model \\
\hline JRC & RANS standard $(k-\varepsilon)$ & Modified Eddy Dissipation Concept \\
\hline TNO & RANS standard $(k-\varepsilon)$ & \\
\hline UU & RNG-LES & Gradient method \\
\hline
\end{tabular}

\subsection{Description of the experiment}

The large-scale deflagration experiment in the open atmosphere was conducted in hemispherical balloon with a radius of $10 \mathrm{~m}$ and a volume of 2094 $m^{3}$. It was filled with stoichiometric hydrogen-air mixture and was fixed to the ground, as shown in Fig. 1 [4]. The mixing produced homogeneous concentration of $29.7 \%$ by volume at the initial temperature and pressure of $283 \mathrm{~K}$ and $98.9 \mathrm{kPa}$ respectively. After combustion, the balloon bursts and the hydrogen-air mixture is mixed with the surrounding air.

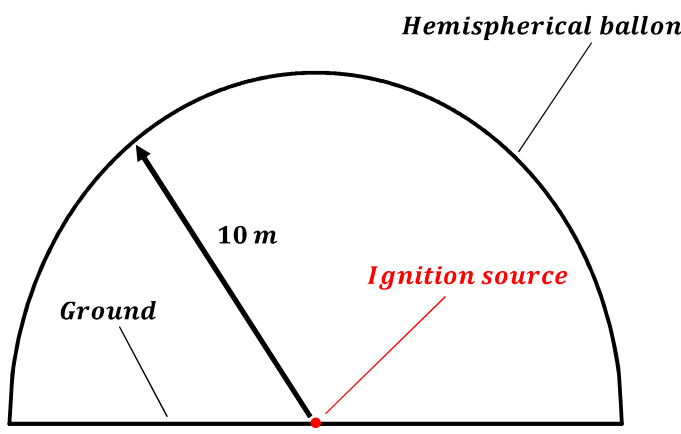

Fig. 1. Schematic of the open atmosphere experiment setup [4].

\subsection{Description of the simulation}

$3 \mathrm{D}$ numerical simulation is performed using ANSYS-Fluent software. The developed model is implemented in user defined function (UDF), which was initially developed at Ulster University. 3D domain with length, width, and height of $200 \mathrm{~m}, 200$ 
$m$, and $100 m$ respectively is used to simulate the experiment.

The hemisphere of radius of $10 \mathrm{~m}$ from the domain center is filled with stoichiometric hydrogen-air mixture, which has volumetric concentration of $29.7 \%$, while the rest of the domain is filled with pure air. The domain ground boundary condition is modelled as an adiabatic wall with no slip. The top and side boundaries of the domain are set to pressure far field, which does not allow reflection of acoustic waves. The simulation initial temperature is set to
$283 K$ and initial pressure is set to 98.9 $k P a$.

\subsection{Results of the simulation}

Fig. 2 shows flame tip propagation radius of the current study compared with the experiment and the state-of-theart (SOTA). Flame radius from the current proposed model is qualitatively compared well with the experiment. Some of the SOTA predicted flame propagation radius close to the experiment while the others predicted slow propagation.

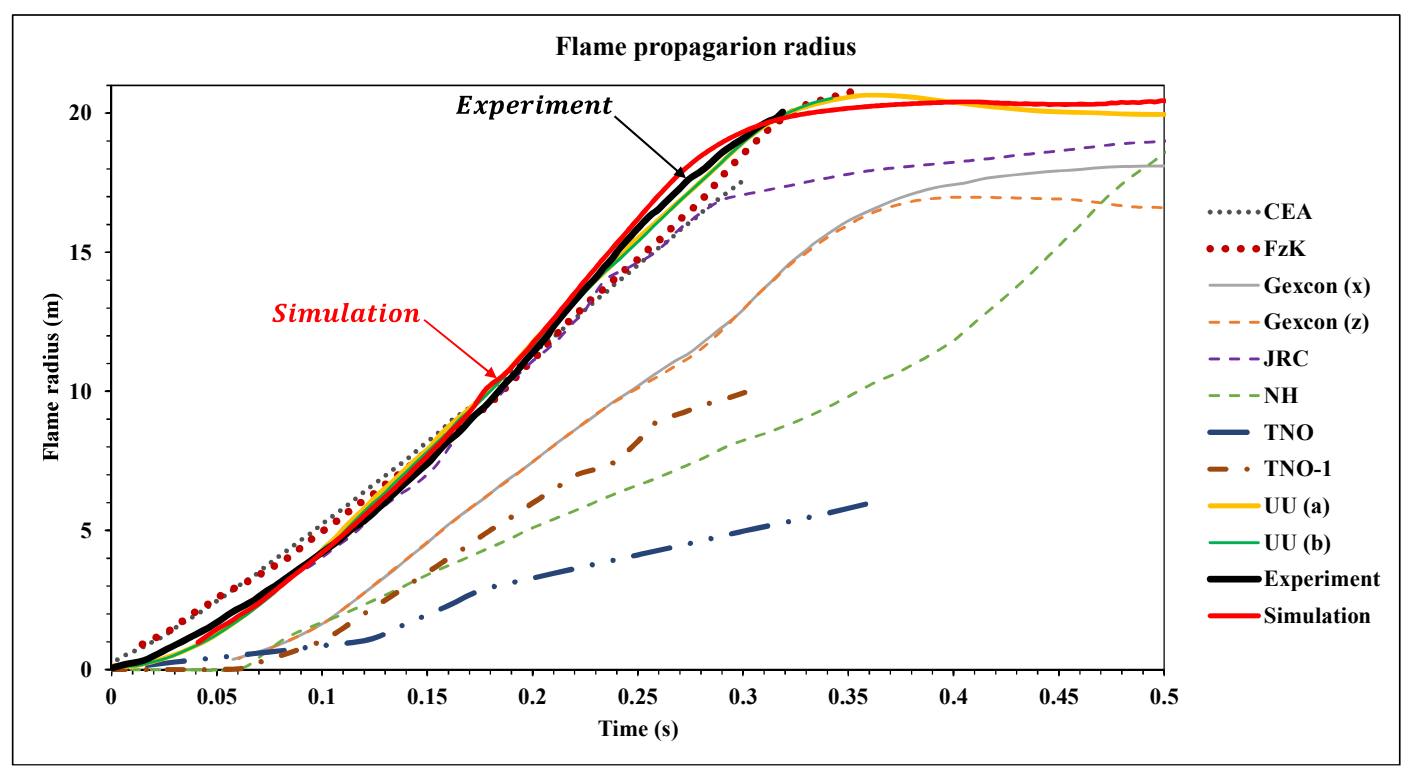

Fig. 2. Flame tip propagation radius for the simulation compared with the experiment and the SOTA [2]

Figs. 3-8 show the overpressure at different transducers from the current simulation compared with the experiment and the SOTA. Some of the overpressure data from the SOTA have high oscillations, which are not considered in the current presentation. Overpressure signals of the experiment are in general smooth, when extracting the experiment data some of the oscillations are removed. 
Pressure transducers inside the flame region were affected by the heat of combustion during the experiment, therefore they did not return to the ambient pressure as shown in Figs. 3, 5, and 6 . The pressure transducer at $5 \mathrm{~m}$ returned to the ambient pressure, because it was thermally protected. The measured overpressure at $2 \mathrm{~m}$ is lower than the measured overpressures at $5 \mathrm{~m}, 8 \mathrm{~m}$, and $18 \mathrm{~m}$. It is also lower than most of the prediction of the SOTA. Therefore, it was assumed that this sensor did not measure the correct overpressure [3]. Hence, the comparison with the experiment's overpressures is qualitatively considered, especially for most of the sensors inside the combustion region.

Pressure dynamics were explained in [2] as follows: overpressures inside the balloon in the unburned mixture in front of the flame were increased due to compression by the thermal expansion and the closure of the balloon. This was followed by a sudden decrease due to the burst of the balloon close to $0.1 \mathrm{~s}$, which was at the flame radius close to $5 \mathrm{~m}$. After that, the overpressures were kept constant until the arrival of the expansion wave, which decreased the overpressures to the negative values, as shown in Figs. 3-5. The expansion wave was generated due to flame quenching. Outside the balloon, the overpressures were increased linearly due to compression wave derived by the thermal expansion. This was followed by a decrease to the negative values due to the expansion wave, as shown in Figs. 6-8.

There are discrepancies between the SOTA and the experiment. The present simulation qualitatively predicts overpressures in the same order of magnitude as the experiment and the SOTA, for both the compression and expansion phases. 


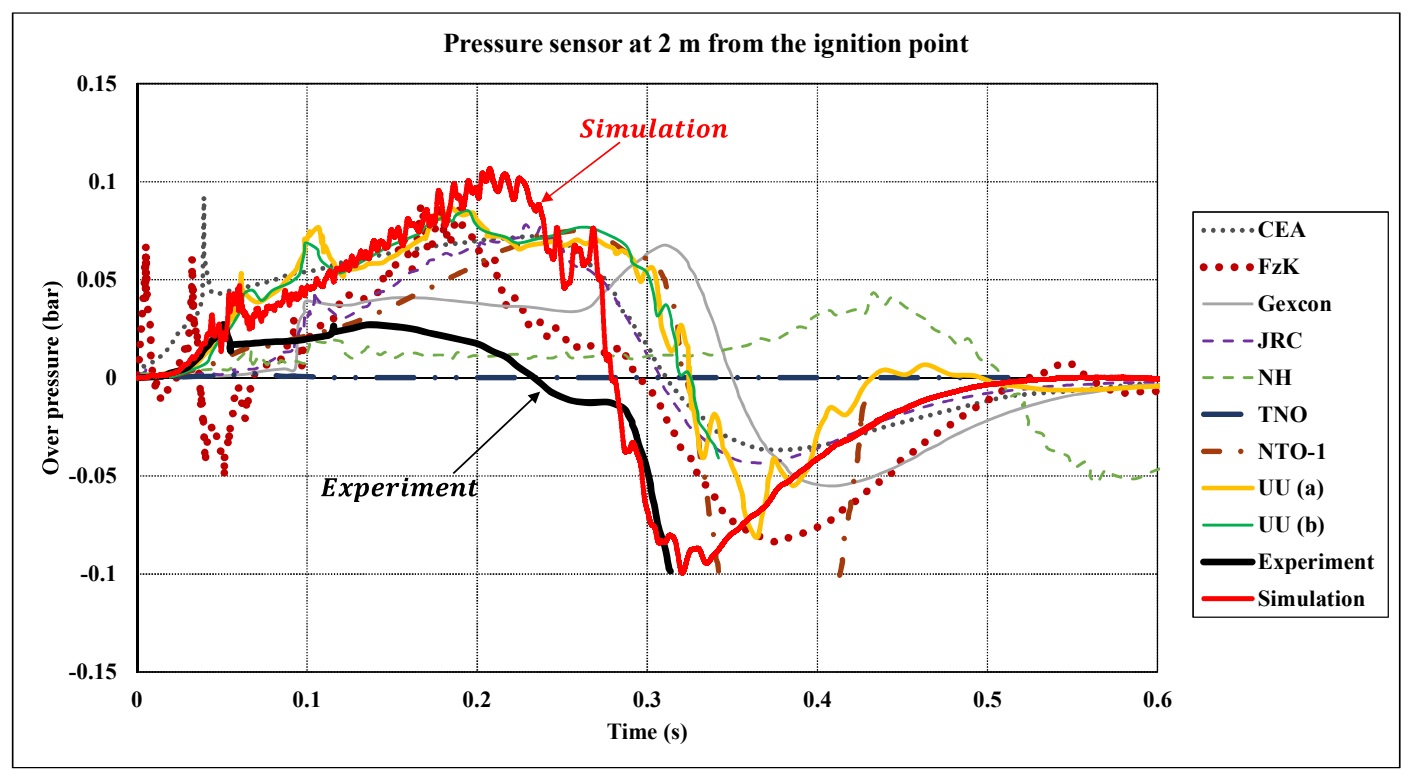

Fig. 3. Overpressure dynamics at $2 m$ compared with the experiment and the SOTA [2].

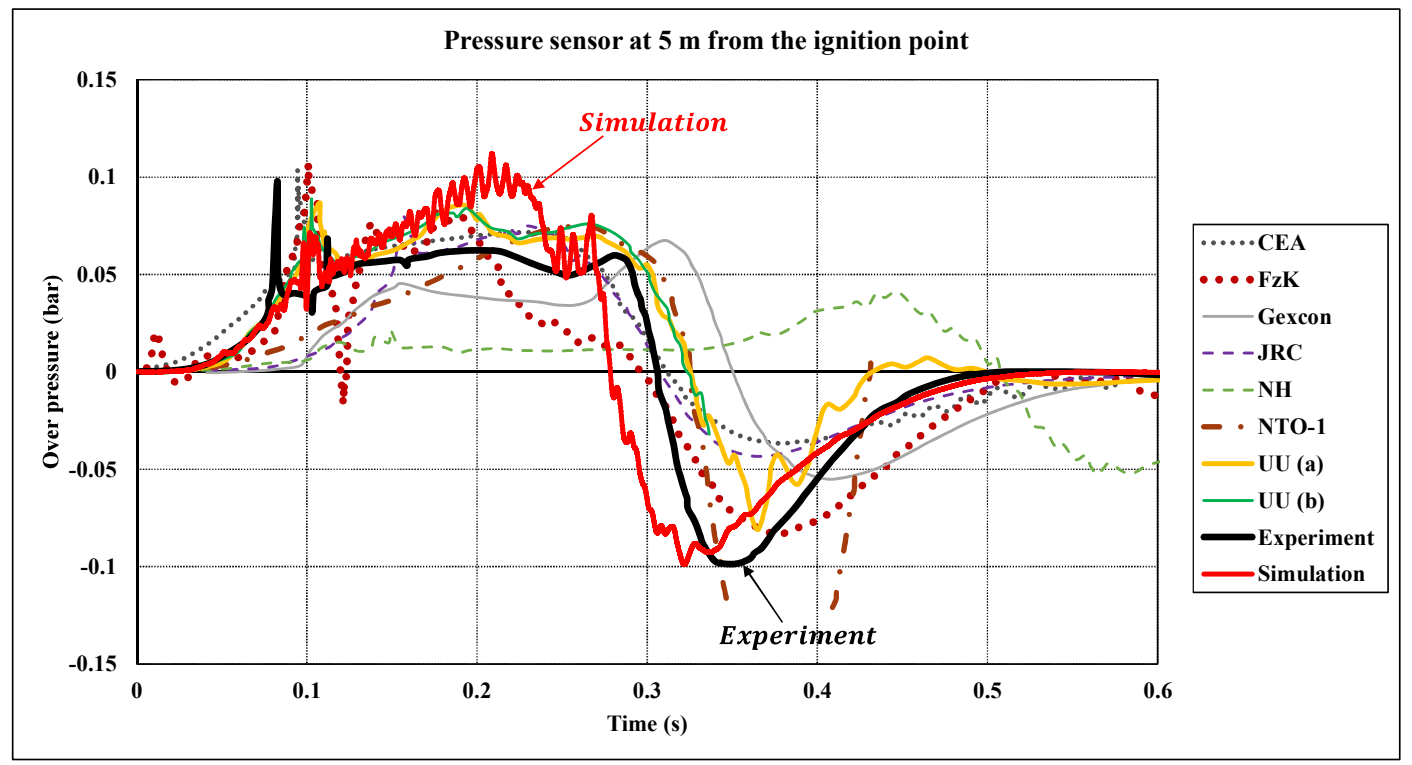

Fig. 4. Overpressure dynamics at $5 m$ compared with the experiment and the SOTA [2]. 


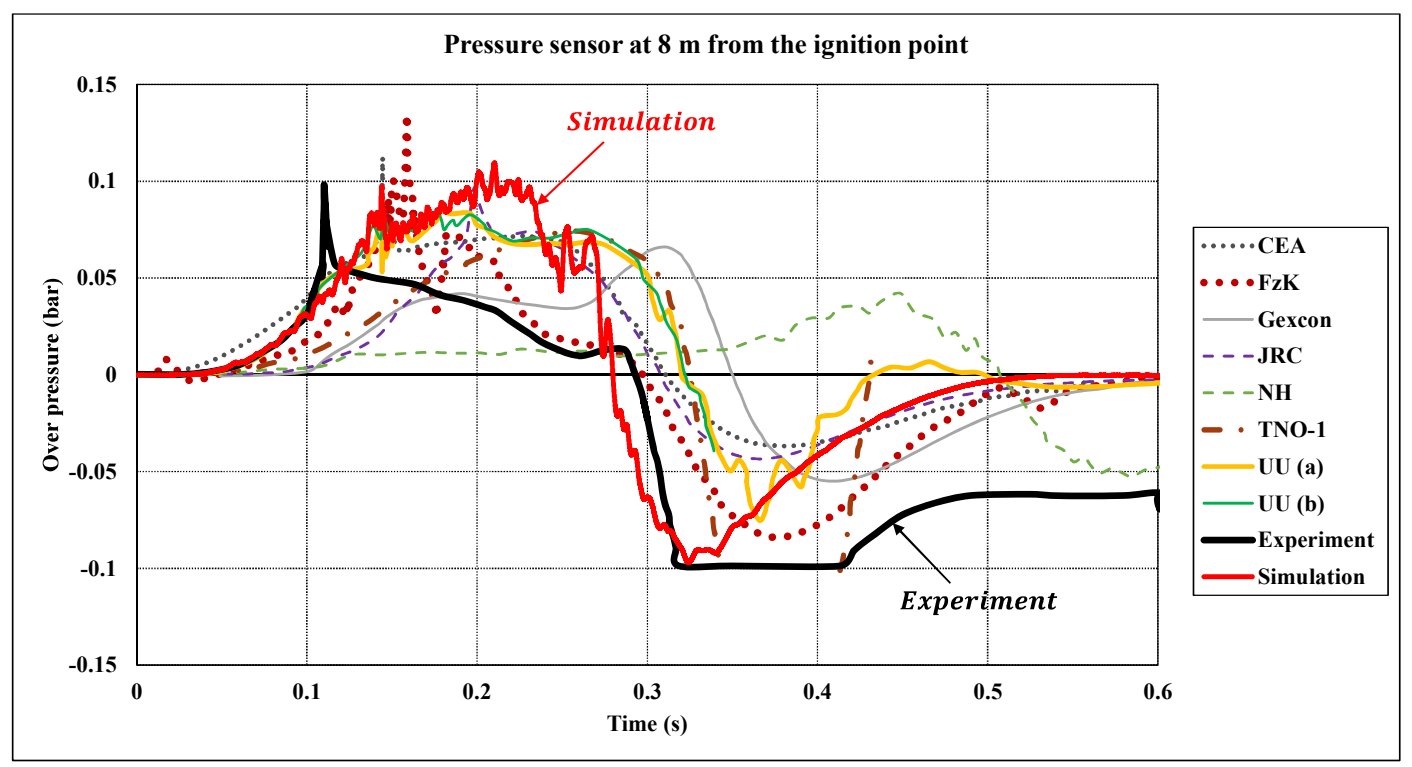

Fig. 5. Overpressure dynamics at $8 m$ compared with the experiment and the SOTA [2].

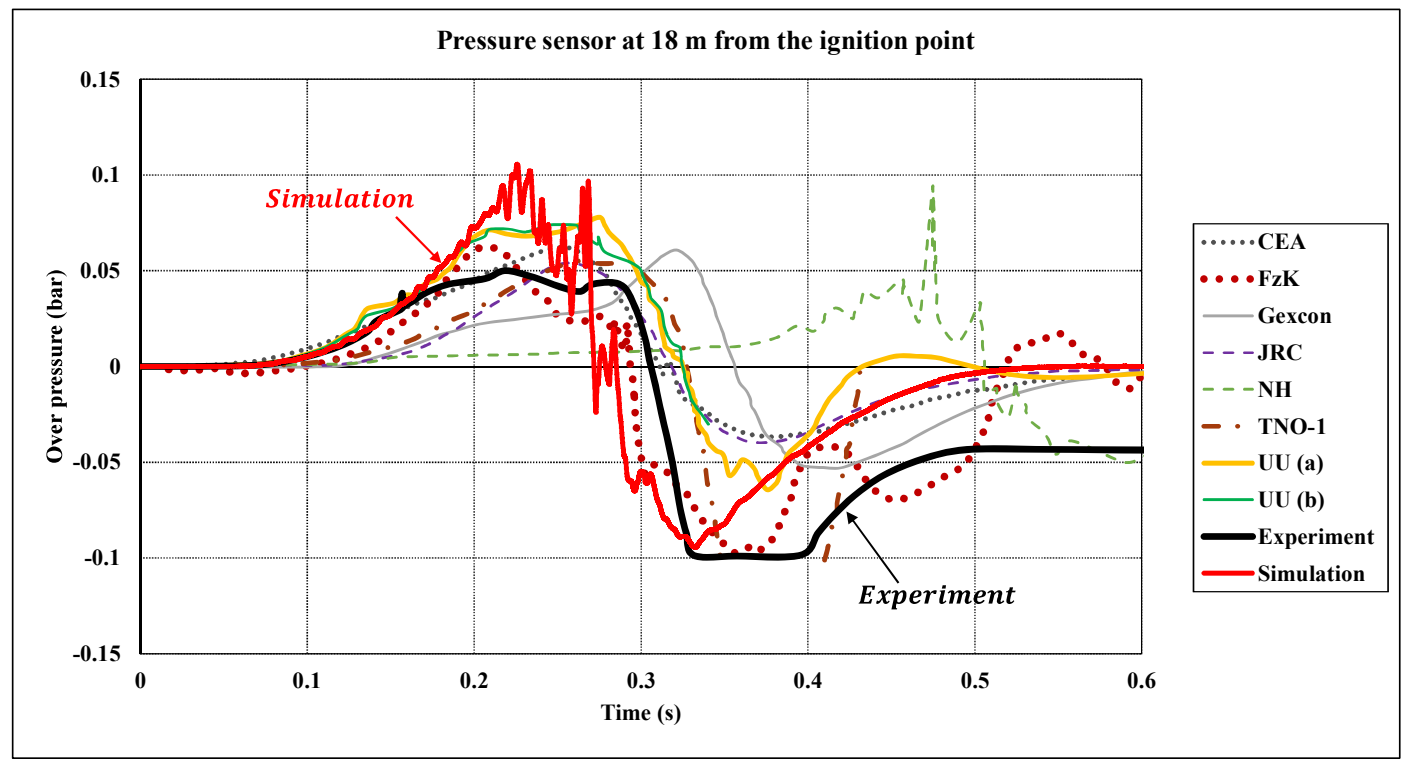

Fig. 6. Overpressure dynamics at $18 \mathrm{~m}$ compared with the experiment and the SOTA 


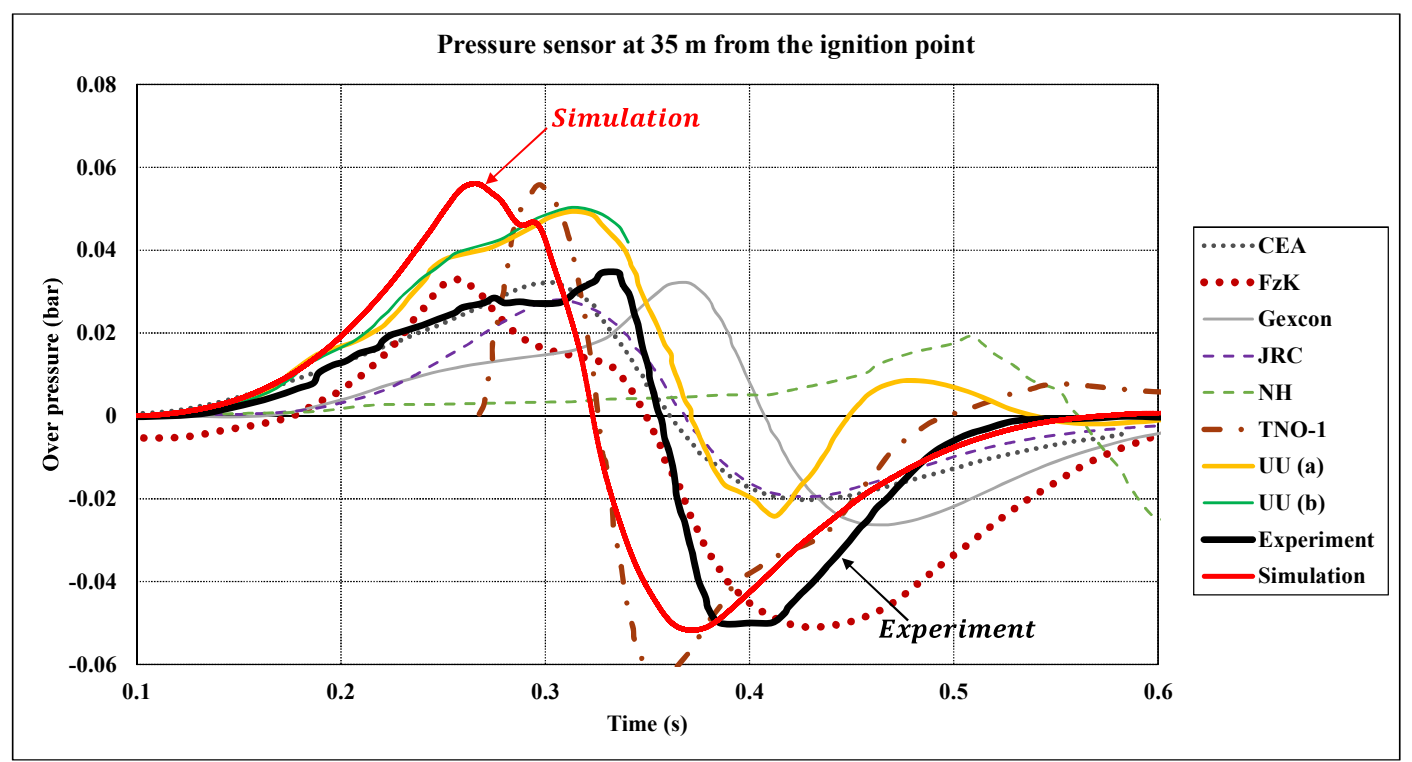

Fig. 7. Overpressure dynamics at $35 \mathrm{~m}$ compared with the experiment and the SOTA

[2].

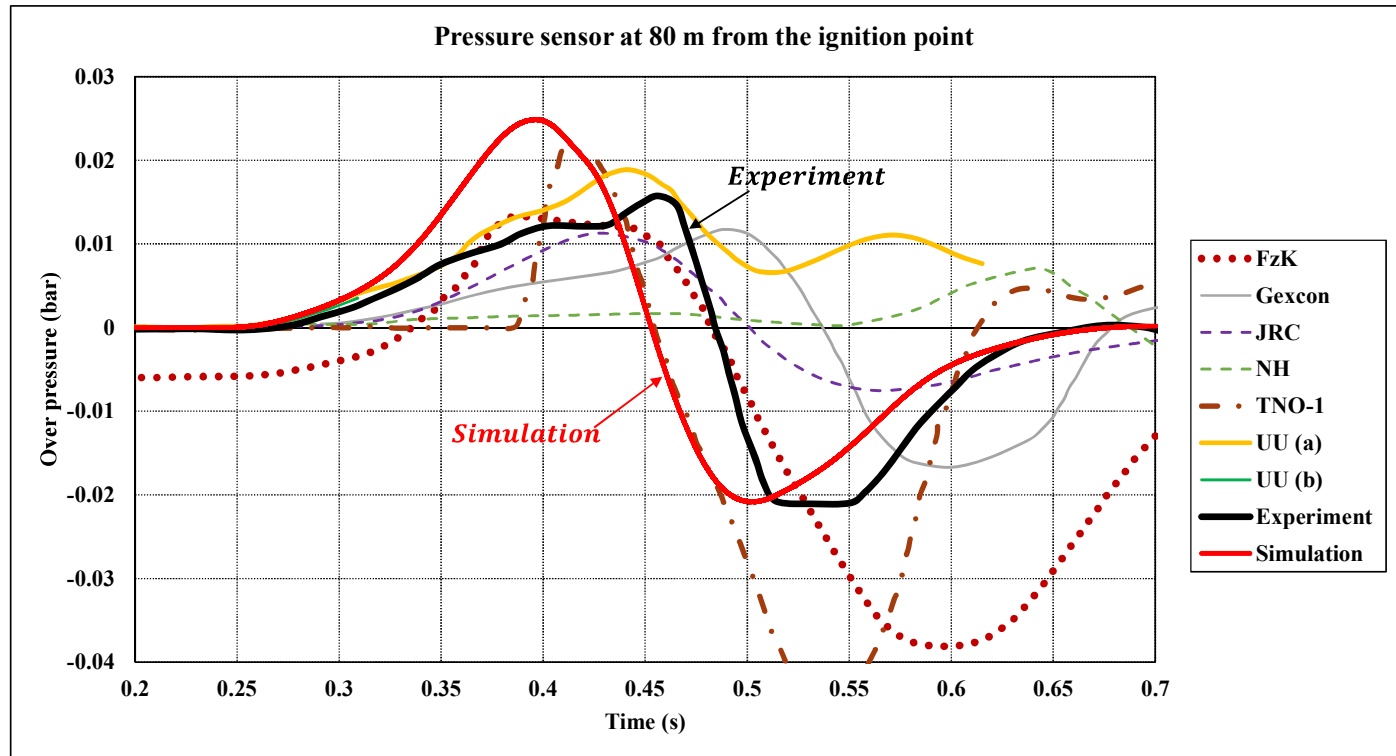

Fig. 8. Overpressure dynamics at $80 \mathrm{~m}$ compared with the experiment and the SOTA

[2].

\section{Conclusions}

Deflagration of hydrogen-air mixture is modelled based on the conservation of the filtered equations of the mass, momentum, energy, and species mass fraction. Turbulence is closed based on the filtered dynamic turbulent kinetic energy equation. Flame-generated turbulence is included as an algebraic term to the momentum equation. Combustion is closed adopting the flame 
acceleration concept. Acceleration mechanisms due to hydrodynamic \& thermo-diffusive instabilities, turbulence, and their interaction are included, in addition to, the possibility of flame quenching due to excessive stretching.

The model is tested against open atmosphere experiment. The predicted flame propagation radius and overpressures are qualitatively comparable with the experiment and the SOTA simulations.

The capability of the model to predict deflagration-to-detonation transition in channel is presented in the future.

\section{Acknowledgments}

I would like to thank Dr. Dmitriy Makarov from Ulster University for discussion and for the technical support. I would like also to thank Dr. Vincent Robin from University of Poitiers France and Dr. Arief Dahoe from TOP BV - Netherlands for their discussion. I acknowledge the funding from Ulster University. Images used courtesy of ANSYS, Inc.

\section{Reference}

[1] M. Sakr, "Modelling and Large Eddy Simulation of Large-scale Hydrogen-air Deflagration and
Deflagration-to-Detonation Transition, [PhD thesis], Ulster University," 2020.

[2] E. Gallego et al., "An Intercomparison Exercise on the Capabilities of CFD Models to Predict Deflagration of a LargeScale H2-Air Mixture in Open Atmosphere-Paper," ICHS, 2005.

[3] J. García et al., "An intercomparison exercise on the capabilities of CFD models to reproduce a large-scale hydrogen deflagration in open atmosphere," Int. J. Hydrogen Energy, vol. 35, no. 9, pp. 44354444, 2010.

[4] T. Jordan, "HySafe project: SBEP-V2: Fh-ICT Balloon Test Deflagration of large-scale stoichiometric hydrogen-air mixture in open atmosphere 20-m diameter hemisphere http://www.hysafe.org/download/ 768/SBEPV2_Spec.pdf," 2006. 